\title{
Satisfacción con la atención recibida en un servicio diferenciado para adolescentes de un establecimiento de salud de primer nivel de atención
}

Danna Maribel Obregón-Morales* 1,a; Giovana Gladys Pante Salas 1,b; John Barja-Ore 1,c; Andrea Mera-Yauri 1,d

\section{RESUMEN}

Objetivo: Determinar la satisfacción de los adolescentes por la atención recibida en un servicio diferenciado de un establecimiento del primer nivel de atención de Lima.

Materiales y métodos: Estudio observacional, descriptivo y transversal realizado en 84 adolescentes atendidos en el Centro de Salud El Progreso (Carabayllo). Se evaluó la satisfacción con la atención recibida a través del cuestionario SERVQUAL, adaptado y validado en contenido, y además confiable en sus componentes de expectativa y percepción.

Resultados: La satisfacción con la atención se presentó en 28,57 \% de adolescentes, en este grupo, la mayoría tenía de 12 a 14 años (83,33\%). En las dimensiones, la empatía y seguridad fueron las que tuvieron mayor porcentaje de satisfacción $(39,28$ y $36,90 \%$, respectivamente). Los indicadores de apariencia física de las instalaciones $(6,45 \pm 0,82)$ y la confianza establecida en la atención $(6,38 \pm 0,84)$ fueron los de mayor puntaje en las expectativas.

Conclusiones: Una proporción menor de adolescentes que asistieron al servicio diferenciado estuvieron satisfechos con la atención recibida, en tanto, la dimensión de empatía fue la que presentó una mayor cantidad de adolescentes con un estado de satisfacción.

Palabras clave: Satisfacción del paciente; Servicios de salud del adolescente; Calidad de atención de salud (Fuente: DeCS BIREME).

\section{Satisfaction from differentiated health care service for adolescents at a primary health care institution}

\section{ABSTRACT}

Objective: To determine adolescent satisfaction from the differentiated health care service at a primary health care institution in Lima.

Materials and methods: An observational, descriptive and cross-sectional study conducted in 84 adolescents treated at the Centro de Salud El Progreso (Carabayllo). Satisfaction from health care was evaluated using the SERVQUAL questionnaire, which was adapted and validated, and showed reliability in its expectation and perception components.

Results: Satisfaction from health care occurred in $28.57 \%$ of the adolescents, most of whom were between 12 and 14 years old $(83.33 \%)$. Regarding the dimensions, empathy and security had the highest percentage of satisfaction (39.28\% and $36.90 \%$, respectively). The indicators physical appearance of the facilities $(6.45 \pm 0.82)$ and trust in health care $(6.38 \pm 0.84)$ presented the highest score in expectations.

Conclusions: A small proportion of adolescents who attended the differentiated service were satisfied with the care received, while the empathy dimension was the one with the greatest number of adolescents who showed satisfaction.

Keywords: Patient satisfaction; Adolescent health service; Quality of health care (Source: MeSH NLM).

1 Universidad Nacional Mayor de San Marcos, Lima, Perú.

a Licenciada en Obstetricia.

b Licenciada en Obstetricia, Magister en Gerencia de Servicio de Salud.

c Licenciado en Obstetricia, Magister en Docencia e Investigación en Salud.

d Bachiller en Obstetricia. 


\section{INTRODUCCIÓN}

La adolescencia es la etapa de vida comprendida entre los 12 y 17 años ${ }^{(1)}$ caracterizada por diversos cambios en todas las áreas del desarrollo humano. Es considerada como un periodo vulnerable a riesgos para la salud, debido a la mayor exposición a todo tipo de violencia, al consumo de alcohol y drogas, a los accidentes de tránsito y problemas de salud mental (2). Además, se enfrentan a situaciones vinculadas a la salud sexual y reproductiva, como el embarazo no deseado y el contagio de infecciones de transmisión sexual ${ }^{(3)}$ que, a su vez, tendrán un efecto a corto o largo plazo.

Estos problemas o condiciones de riesgo pueden variar según los contextos sociales, económicos y culturales en los que los adolescentes se desarrollan (4), lo cual devela la necesidad de fomentar el cuidado y orientación en este grupo y, sobre todo, generar oportunidades de desarrollo integral, a través de la implementación de entornos que garanticen sus derechos, como el acceso a servicios de salud diferenciados y de calidad ${ }^{(4,5)}$.

Según recomienda la Organización Mundial de la Salud (OMS), estos servicios deben ser accesibles, aceptables, equitativos, apropiados y efectivos ${ }^{(5)}$; no obstante, algunos estudios evidencian que todavía existen brechas relevantes en su adecuada prestación, ya que aún no se disponen de características esenciales básicas como un horario diferenciado, falta de privacidad y confidencialidad de la información, y la presencia de un personal de salud multidisciplinario con competencias específicas para brindar esta atención ${ }^{(6,7)}$.

Asimismo, se ha reconocido como un punto crítico la ausencia del trabajo colaborativo con el adolescente en el desarrollo de prioridades y mejoras en la calidad de dichos servicios ${ }^{(8)}$, por lo que su evaluación no solo debe considerar aspectos técnicos ${ }^{(9)}$, sino también la perspectiva del adolescente y su satisfacción, entendida como la brecha entre lo que espera recibir en la atención y lo que percibe de la misma y, a partir de ello, mejorar la fidelización a estos servicios ${ }^{(10)}$.

En el Perú, las necesidades de los adolescentes están marcadas por diferentes condiciones sociales como la pobreza, baja escolaridad e inadecuado acceso a servicios de salud, que a su vez pueden verse más afectadas por factores como el sexo, orientación sexual y otras condiciones de discriminación que afecten su desarrollo ${ }^{(11,12)}$. En este marco, el Ministerio de Salud (MINSA) implementó políticas públicas para prestar atención integral en servicios diferenciados y con equipos interdisciplinarios.

La implementación de estos servicios requiere de estrategias de descentralización, coordinación y participación de los adolescentes, la familia y la comunidad.
Esta ejecución debe regirse por los enfoques de derecho (incluido el derecho sexual y reproductivo), el género, la diversidad, la interculturalidad, la intergeneracionalidad y ser de carácter preventivo-promocional, por lo que cada establecimiento deberá organizar el servicio con base en los recursos disponibles y el nivel de complejidad, ya sea dentro o fuera de estos, como los colegios u otros lugares, con el fin de acercar el acceso de salud al adolescente ${ }^{(13)}$.

Pese a la implementación de estas políticas, la participación de los sectores involucrados aún es insuficiente ${ }^{(14)}$, lo que se evidencia en la falta de espacios exclusivos para la atención del adolescente, personal no capacitado, escasa disponibilidad de materiales y equipos adecuados, reducido número de atenciones, horarios no diferenciados y estrategias poco pertinentes a las necesidades de los adolescentes (15); lo que, en conjunto, afecta directamente la calidad de la prestación del servicio.

Por lo expuesto, este estudio se desarrolló con el objetivo determinar la satisfacción en la atención recibida por los adolescentes en un servicio diferenciado de un establecimiento del primer nivel de atención de Lima.

\section{MATERIALES Y MÉTODOS}

\section{Diseño y población}

El estudio fue de enfoque cuantitativo, de diseño observacional, descriptivo y transversal. La población estuvo conformada por 92 adolescentes de 12 a 17 años, quienes fueron atendidos en un servicio diferenciado del Centro de Salud El Progreso de Carabayllo, durante los meses de enero y febrero del 2020. El tamaño de la muestra fue de 84 adolescentes, quienes fueron seleccionados por un muestreo no probabilístico, por conveniencia.

Se incluyeron a adolescentes varones y mujeres que alcanzaron una cita para su atención y a aquellos que decidieron participar voluntariamente con la autorización de sus padres. Las adolescentes embarazadas y los que no completaron el instrumento de recolección de datos o presentaron inconsistencias fueron excluidos de la investigación.

\section{Variables y mediciones}

La recolección de datos se realizó a través de la técnica de la encuesta autoadministrada, y utilizando un cuestionario SERVQUAL basado en la Guía Técnica para la Evaluación de la Satisfacción del Usuario Externo en los Establecimientos y Servicios Médicos de Apoyo del MINSA ${ }^{(16)}$. Dicho instrumento fue adaptado y validado en contenido por tres expertas temáticas con amplia experiencia en la prestación de los servicios diferenciados para adolescentes. El cuestionario se organizó en dos partes: en la primera se recababa información de la edad, sexo y tipo de usuario; y la segunda parte tuvo dos componentes para la medición de la variable 
en estudio: el de Expectativas (E), que se aplicó antes de recibir la atención; y el de Percepciones $(P)$, posterior a esta. Cada componente estaba conformado por 23 enunciados, distribuidos en cinco dimensiones: fiabilidad (1 al 5), capacidad de respuesta (6 al 9), seguridad (10 al 13), empatía (14 al 18), y aspectos tangibles (19 al 23). Cada enunciado fue valorado en una escala Likert, en la que el puntaje más bajo era 1 y el más alto, 7 . Se realizó la sumatoria de puntos de los enunciados de acuerdo a la conformación de las dimensiones y de manera global; con dicha sumatoria en ambos componentes, se consideró como "satisfecho" cuando la diferencia entre percepciones y expectativas fue positiva, e "insatisfecho" cuando fue negativa. Cabe precisar que se realizó una prueba piloto, con la cual se determinó que los componentes Expectativa $(\alpha=0,940)$ y Percepciones $(\alpha=0,930)$ poseían una elevada confiabilidad.

\section{Análisis estadístico}

Los datos fueron registrados en una matriz elaborada en el programa Microsoft Excel 2016, la cual fue exportada para su procesamiento al software SPSS versión 26 . Para las variables categóricas se estimaron frecuencias absolutas y relativas; y para las numéricas, la media y desviación estándar. Como método estadístico se seleccionó la prueba exacta de Fisher para establecer diferencias entre las categorías de las variables.

\section{Consideraciones éticas}

La investigación fue aprobada por el Comité de Ética de la Facultad de Medicina de la Universidad Nacional Mayor de San Marcos, y contó con la respectiva aprobación institucional para su ejecución. Además, se empleó el asentimiento informado, para los adolescentes, y el consentimiento informado, para sus padres, lo que aseguró la participación voluntaria. Asimismo, se resguarda la confidencialidad de la información, ya que solo fue utilizada para los fines del estudio.

\section{RESULTADOS}

Los hallazgos del estudio demostraron que $28,57 \%$ de los adolescentes estuvo satisfecho con la atención recibida en el servicio diferenciado. En tanto, en la tabla 1 se detalla que la proporción de adolescentes de 12 a 14 años fue mayor en el grupo de los que estuvieron satisfechos en comparación a aquellos que no $(83,33 \%$ vs. $58,33 \%)$, con diferencias significativas entre estos grupos $(p=0,041)$. Además, se muestra que la mayoría de adolescentes satisfechos e insatisfechos fueron mujeres y usuarios nuevos en el servicio.

Tabla 1. Satisfacción con la atención recibida según las características generales de los adolescentes

\begin{tabular}{|c|c|c|c|c|c|}
\hline \multirow[t]{2}{*}{ Características generales } & \multicolumn{2}{|c|}{ Satisfecho } & \multicolumn{2}{|c|}{ Insatisfecho } & \multirow[b]{2}{*}{$p^{*}$} \\
\hline & $\mathrm{n}$ & $\%$ & $\mathrm{n}$ & $\%$ & \\
\hline \multicolumn{6}{|l|}{ Edad } \\
\hline 12 a 14 años & 20 & 83,33 & 35 & 58,33 & \\
\hline 15 a 17 años & 4 & 16,67 & 25 & 41,67 & 0,041 \\
\hline \multicolumn{6}{|l|}{ Sexo } \\
\hline Masculino & 8 & 33,33 & 22 & 36,67 & \\
\hline Femenino & 16 & 66,67 & 38 & 63,33 & 0,807 \\
\hline \multicolumn{6}{|l|}{ Tipo de usuario } \\
\hline Nuevo & 17 & 70,83 & 43 & 71,67 & \\
\hline Continuador & 7 & 29,17 & 17 & 28,33 & 1,000 \\
\hline Total & 24 & 100 & 60 & 100 & \\
\hline
\end{tabular}

* $p$ valor estimado para la prueba exacta de Fisher

En la figura 1 se observa una tendencia a la insatisfacción con la atención recibida en cada una de las dimensiones evaluadas. La fiabilidad $(76,20 \%)$ y los aspectos tangibles $(67,86 \%)$ son las que se señalaron con mayor frecuencia como fuente de insatisfacción; mientras que la dimensión empatía presentó un mayor número de adolescentes satisfechos con la atención $(39,28 \%)$. 


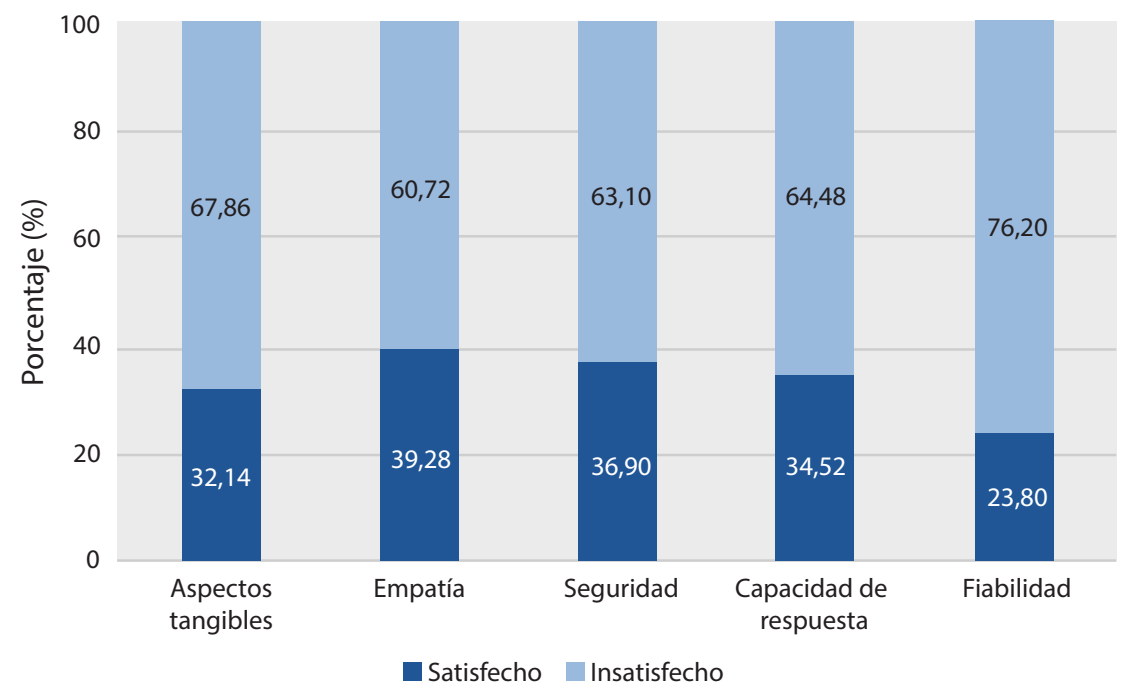

Figura 1. Satisfacción con la atención recibida, según sus dimensiones, en los adolescentes

El análisis de los indicadores de las dimensiones en la tabla 2 muestra que, en la mayoría de casos, la expectativa hacia la atención fue mayor que la percepción que se tuvo; sobre esto, la apariencia física de las instalaciones $(6,45 \pm 0,82)$ y la confianza establecida en la atención $(6,38 \pm 0,84)$ fueron las que presentaron mayor expectativa por parte de los adolescentes. Por otro lado, la disponibilidad de los servicios después de la atención fue la mejor valorada desde la percepción de los usuarios $(6,38 \pm 0,91)$, seguida de indicadores como la confidencialidad $(6,32 \pm 0,99)$, personal capacitado $(6,32 \pm 0,92)$ e interés de necesidades $(6,32 \pm 1,03)$. En tanto, el tiempo de espera $(4,68 \pm 1,76)$ y la atención en un horario conveniente $(4,93 \pm 1,62)$ fueron los indicadores con menor valoración.

Tabla 2. Expectativas y percepciones sobre los indicadores de las dimensiones de la satisfacción con la atención recibida

Dimensiones / Indicadores

\section{Fiabilidad}

Horario conveniente

Programación por orden de llegada

Disponibilidad de información

Atención en salud sexual y reproductiva

Orientación del personal

Capacidad de respuesta

Señalizaciones y avisos

Tiempo de respuesta

Tiempo de consulta

Disponibilidad de servicios

\section{Seguridad}

Ambiente privado y limpio

Confidencialidad

Confianza

Personal capacitado

$\begin{array}{ll}5,86 \pm 1,29 & 4,93 \pm 1,62 \\ 6,26 \pm 1,12 & 5,31 \pm 1,72 \\ 6,19 \pm 1,15 & 5,94 \pm 0,98 \\ 6,11 \pm 1,17 & 6,07 \pm 1,18 \\ 6,35 \pm 0,73 & 5,95 \pm 1,04 \\ & \\ 5,80 \pm 1,31 & 5,67 \pm 1,25 \\ 5,85 \pm 1,24 & 4,68 \pm 1,76 \\ 6,00 \pm 1,16 & 5,73 \pm 1,28 \\ 6,21 \pm 0,99 & 6,38 \pm 0,91\end{array}$
$6,60 \pm 0,69$
$6,36 \pm 0,87$
$6,26 \pm 1,13$
$6,32 \pm 0,99$
$6,38 \pm 0,84$
$6,30 \pm 0,94$
$6,25 \pm 1,04$
$6,32 \pm 0,92$ 


\section{Dimensiones / Indicadores}

\section{Empatía}

Comprensión de necesidades

$6,11 \pm 0,87$

$6,20 \pm 0,88$

Uso de lenguaje claro y comprensible

Interés de necesidad

Personal disponible

Cortesía y amabilidad

Aspectos tangibles

Comodidad

Apariencia física del equipo de atención

Apariencia física de instalaciones

Disponibilidad de materiales y equipos

Uso de tecnología

\section{Expectativa $(\bar{x} \pm D . E)$}

\section{Percepción $(\overline{\mathrm{x}} \pm \mathrm{D} . \mathrm{E})$}

$6,25 \pm 0,91 \quad 6,32 \pm 1,03$

$6,14 \pm 0,97 \quad 5,86 \pm 0,94$

$6,52 \pm 0,96 \quad 6,37 \pm 0,80$

$\begin{array}{ll}6,26 \pm 1,11 & 6,10 \pm 1,30 \\ 6,25 \pm 0,90 & 6,24 \pm 1,01 \\ 6,45 \pm 0,82 & 6,21 \pm 0,87 \\ 6,37 \pm 0,92 & 5,98 \pm 1,08 \\ 6,24 \pm 0,95 & 5,56 \pm 1,32\end{array}$

La distribución de las características generales de los adolescentes según su estado de satisfacción en cada dimensión se aprecia en la tabla 3. Al respecto, resalta que entre aquellos adolescentes satisfechos e insatisfechos en cada una de las dimensiones, la mayoría tenía de 12 a 14 años de edad, eran de sexo femenino y eran usuarios nuevos en el servicio, lo que demuestra que no existen diferencias significativas en todos los grupos de análisis $(p>0,05)$.

Tabla 3. Satisfacción con la atención recibida en las dimensiones, según las características generales de los adolescentes

\begin{tabular}{|c|c|c|c|c|c|c|c|c|c|}
\hline \multirow[t]{3}{*}{ Dimensiones } & \multicolumn{3}{|c|}{ Edad } & & \multicolumn{4}{|c|}{ Sexo } & \multirow{3}{*}{$P$} \\
\hline & 12 a 14 años & \multicolumn{2}{|c|}{15 a 17 años } & \multirow[t]{2}{*}{$P$} & \multicolumn{2}{|c|}{ Masculino } & \multicolumn{2}{|c|}{ Femenino } & \\
\hline & $\%$ & $\mathrm{n}$ & $\%$ & & $\mathrm{n}$ & $\%$ & $\mathrm{n}$ & $\%$ & \\
\hline
\end{tabular}

Fiabilidad

Satisfecho

1680,00

Insatisfecho

3960,93

420,00

0,178

$7 \quad 35,00$

13

65,00

Capacidad de respuesta

Satisfecho

Insatisfecho

$\begin{array}{llll}20 & 68,96 & 9 & 31,04\end{array}$

$35 \quad 63,63 \quad 20$

36,36

0,810

11

35,93

41

$64,07 \quad 1,000$

Seguridad

Satisfecho
Insatisfecho

$23 \quad 74,19$

\section{Empatía}

Satisfecho

32

60,37

8

25,81

0,240

10

32,25

37,93

18

62,06

Insatisfecho

$\begin{array}{ccc}24 & 72,72 & 9 \\ 31 & 60,78 & 20\end{array}$

27,28

39,22

0,348

$11 \quad 33,33$

22

$19 \quad 37,25$

32

66,67

Aspectos tangibles

Satisfecho
Insatisfecho

$\begin{array}{ccc}18 & 66,67 & 9 \\ 37 & 64,91 & 20\end{array}$

33,33
35,09

1,000

11

40,74

16

59,26

$19 \quad 33,33 \quad 38$

66,67 


\begin{tabular}{|c|c|c|c|c|c|}
\hline \multirow[t]{3}{*}{ Dimensiones } & \multicolumn{4}{|c|}{ Tipo de usuario } & \multirow{3}{*}{$P$} \\
\hline & \multicolumn{2}{|c|}{ Nuevo } & \multicolumn{2}{|c|}{ Continuador } & \\
\hline & $\mathrm{n}$ & $\%$ & $\mathrm{n}$ & $\%$ & \\
\hline \multicolumn{6}{|l|}{ Fiabilidad } \\
\hline Satisfecho & 16 & 80,00 & 4 & 20,00 & \\
\hline Insatisfecho & 44 & 68,75 & 20 & 31,25 & 0,405 \\
\hline \multicolumn{6}{|c|}{ Capacidad de respuesta } \\
\hline Satisfecho & 19 & 65,51 & 10 & 34,49 & \\
\hline Insatisfecho & 41 & 74,54 & 14 & 25,46 & 0,450 \\
\hline \multicolumn{6}{|l|}{ Seguridad } \\
\hline Satisfecho & 21 & 67,75 & 10 & 32,25 & \\
\hline Insatisfecho & 39 & 73,58 & 14 & 26,42 & 0,621 \\
\hline \multicolumn{6}{|l|}{ Empatía } \\
\hline Satisfecho & 24 & 72,72 & 9 & 27,28 & \\
\hline Insatisfecho & 36 & 70,58 & 15 & 29,42 & 1,000 \\
\hline \multicolumn{6}{|c|}{ Aspectos tangibles } \\
\hline Satisfecho & 22 & 81,48 & 5 & 18,52 & \\
\hline Insatisfecho & 38 & 66,67 & 19 & 33,33 & 0,201 \\
\hline
\end{tabular}

\section{DISCUSIÓN}

En el proceso de la prestación de un servicio de salud, un indicador clave de la mejora continua de la calidad del mismo es la satisfacción del usuario con la atención que recibe; sobre todo, cuando este usuario tiene diversas expectativas y necesidades propias de su desarrollo, como ocurre en la adolescencia, etapa en la que requiere de un espacio oportuno dentro del sistema sanitario para brindarle una orientación integral dirigida a mejorar su estado de salud y, con ello, lograr una adecuada calidad de vida.

Los resultados de este estudio muestran que el $28,57 \%$ de los adolescentes estuvo satisfecho con la atención recibida. Este hallazgo difiere de la investigación de Mulugeta et al. ${ }^{(7)}$, en la que el 49,10 \% de adolescentes informaron estar satisfechos con el servicio, y de otro estudio realizado en Etiopía ${ }^{(17)}$, en el que el 60,70\% de adolescentes atendidos tuvieron este mismo grado de satisfacción. El nivel de implementación de las normas o protocolos para prestar los servicios estudiados en cada país y la capacidad de adaptación de los mismos a los requerimientos en esta etapa de vida podrían justificar el contraste entre estos resultados. Es evidente la necesidad de reevaluar y reestructurar los procesos que intervienen en la atención.

Asimismo, es preciso señalar que la proporción de adolescentes satisfechos fue mayor en el grupo etario de 12 a 14 años, en comparación al de 15 a 17 años. Este hecho puede ser una muestra de que este grupo de adolescentes tienen menos expectativas o están más predispuestos a participar y recibir de forma propositiva la atención que se les brinda. Esta afirmación supondría, además, un aspecto clave para generar un sentido de pertinencia con el servicio desde edades tempranas y, con ello, asegurar la continuidad de su asistencia y el cumplimiento de actividades planificadas para asegurar el bienestar físico, psicológico y social del adolescente.

Un estudio realizado en Indonesia ${ }^{(18)}$ reportó que un amplio porcentaje de adolescentes está satisfecho respecto a la prestación del servicio en cada una de las dimensiones evaluadas: empatía ( $84 \%$ ) y seguridad ( $82 \%$ ). En Lima, otra investigación ${ }^{(19)}$ señaló que las dimensiones de aspectos tangibles (76\%) y empatía (66\%) fueron las que presentaron más adolescentes satisfechos. Al respecto, el presente trabajo muestra porcentajes menores de satisfacción en las dimensiones señaladas. Con esta información es evidente que existe una tendencia a considerar la relación establecida con el personal de salud encargado de prestar el servicio como uno de los aspectos más relevantes, lo que podría ser determinante para fidelizar al adolescente y mantener su asistencia al establecimiento durante el periodo que lo requiera. Con ello, se demuestra el papel clave del personal y todas las competencias específicas necesarias que debe poseer para atender a este grupo de 
personas.

La investigación realizada por Awang $\mathrm{H}$. et al. (20) demostró una mayor satisfacción respecto a la sensación de comodidad y confianza establecida con el profesional que brindó la atención. Por el contrario, nuestro estudio evidenció que las expectativas sobre la confianza con el personal fueron mayores que las percepciones hacia este indicador. Otros parámetros relevantes y acordes a los cambios sociales fueron el uso de la tecnología, del equipo y los materiales adecuados en el proceso de atención, que mostraron puntajes bajos en su percepción. Este hallazgo podría reflejar que la capacidad de adaptabilidad de los servicios dirigidos a poblaciones específicas es insuficiente. Es posible que la razón sea multifactorial y abarque situaciones como la falta de presupuesto o inversión, poca capacidad de gestión, escasa identificación con el servicio por parte del personal, falta de interés para involucrar a los adolescentes en la estructuración del proceso de atención, entre otros.

Como refieren Chandra-Mouli et al. (21), tanto la implementación de estándares mínimos de calidad, y de pautas claras para lograrlos de manera eficaz y eficiente, son fundamentales para el éxito y cumplimiento de los objetivos propuestos en el proceso de prestación de un servicio de salud; sobre todo, frente a una situación como la que presentamos en esta investigación, donde las expectativas de los adolescentes, en la mayor de parte de indicadores evaluados, superaron a su percepción, y evidenciaron una brecha importante en términos de gestión de calidad que debe ser abordada de forma integral por todos los agentes involucrados en dicho proceso.

Entre las limitaciones de la investigación se precisa que los resultados no pueden ser generalizados, dado el tipo de muestreo empleado; además, ya que la población estudiada proviene de un solo establecimiento de salud, las estimaciones no representan la realidad de los servicios diferenciados en el Perú. De otro lado, debido al diseño descriptivo del estudio, no fue factible realizar comparaciones del estado de satisfacción en los adolescentes usuarios de servicios diferenciados y tradicionales.

Se concluye que el mayor número de adolescentes estuvo insatisfecho con la atención recibida en el servicio diferenciado del establecimiento de salud. Las dimensiones fiabilidad y aspectos tangibles fueron las que presentaron mayor cantidad de adolescentes insatisfechos. Por ello, se sugiere la implementación de estrategias continuas y sostenidas en el tiempo que fortalezcan la planificación, organización y ejecución del proceso de atención en el servicio, considerando las necesidades específicas de los adolescentes.

Contribuciones de los autores: Danna Maribel Obregón-
Morales: diseño de la investigación, recolección de datos, redacción del manuscrito y aprobación de la versión final. Giovana Gladys Pante Salas: diseño de la investigación, redacción del manuscrito y aprobación de la versión final. John Barja-Ore: procesamiento estadístico de los datos, análisis e interpretación de la información, redacción del manuscrito y aprobación de la versión final. Andrea MeraYauri: análisis e interpretación de la información, redacción del manuscrito y aprobación de la versión final.

Fuentes de financiamiento: Este artículo ha sido financiado por los autores.

Conflicto de interés: Los autores declaran no tener conflicto de intereses.

\section{REFERENCIAS BIBLIOGRÁFICAS}

1. Ministerio de Salud del Perú. Norma técnica de salud para la atención integral de salud en la etapa de vida adolescente [Internet]. 2012. Disponible en: http://spij.minjus.gob.pe/Graficos/Peru/2012/ Diciembre/09/RM-973-2012-MINSA.pdf

2. Organización Mundial de la Salud. Estrategia Mundial para la Salud de la Mujer, el Niño y el adolescente (2016-2030) [Internet]. 2015. Disponible en: https://www.who.int/maternal_child_adolescent/ documents/estrategia-mundial-mujer-nino-adolescente-2016-2030. pdf?ua $=1$

3. Organización Mundial de la Salud. Recomendaciones de la OMS sobre salud y derechos sexuales y reproductivos de los adolescentes [Internet]. 2019. Disponible en: https://apps.who.int/iris/bitstream/ handle/10665/312341/9789243514604-spa.pdf?ua=1

4. Liang M, Simelane S, Fortuny G, Michielsen K, Claire D, Snow R. The state of adolescent sexual and reproductive health. J Adolesc Health. 2019; 65(6): S3-15.

5. Organización Mundial de la Salud. Normas mundiales para mejorar la calidad de los servicios de atención de salud de los adolescentes [Internet]. 2016. Disponible en: https://iris.paho. org/bitstream/handle/10665.2/28569/9789275319048_v1-spa. pdf?sequence=1\&isAllowed=y

6. Munea AM, Alene GD, Debelew, G. Quality of youth friendly sexual and reproductive health services in west Gojjam Zone, north West Ethiopia: with special reference to the application of the Donabedian model. BMC Health Serv Res. 2020; 20(1): 245.

7. Mulugeta B, Girma M, Kejela G, Meskel FG, Andarge E, Zerihun E. Assessment of youth-friendly service quality and associated factors at Public Health Facilities in Southern Ethiopia: a facility-based crosssectional study. Biomed Res Int. 2019; 2019: 1-11.

8. Ambresin A, Bennett K, Patton G, Sanci L, Sawyer S. Assessment of youth-friendly health care: a systematic review of indicators drawn from young people's perspectives. J Adolesc Health. 2013; 52(6): 670-81.

9. Vargas V, Valecillos J, Hernández C. Calidad en la prestación de servicios de salud: Parámetros de medición. Rev Cienc Soc. 2013; 19(4): 663-71.

10. Mohamed B, Azizan NA. Perceived service quality's effect on patient satisfaction and behavioural compliance. Int J Health Care Qual Assur. 2015; 28(3): 300-14.

11. Fondo de Población de las Naciones Unidas. Gasto público social en adolescencia y juventud, 2012-2018 [Internet]. 2020. Disponible en: https://peru.unfpa.org/sites/default/files/pub-pdf/peru_informe_ pais_gpsaj_20200721x.pdf

12. Ministerio de Salud del Perú. Situación de Salud de los Adolescentes 
y Jóvenes en el Perú [Internet]. 2017. Disponible en: http://bvs. minsa.gob.pe/local/MINSA/4143.pdf

13. Ministerio de Salud del Perú. Norma técnica de salud para la atención integral de salud de adolescentes [Internet]. 2019. Disponible en: https://www.unicef.org/peru/media/7096/file/ Norma\%20t\%C3\%A9cnica\%20de\%20atenci\%C3\%B3n\%20de\%20salud\%20 adolescente.pdf

14. Mesa de Concertación para la Lucha contra la Pobreza. Embarazo en adolescentes peruanas aumentó [Internet]. 2018. Disponible en: https://www.mesadeconcertacion.org.pe/sites/default/files/ archivos/2018/documentos/06/alerta_embarazo_en_adolescentes_ aumento_junio_2018.pdf

15. Defensoría del Pueblo del Perú. Resultados de supervisión defensorial a servicios de salud diferenciados para adolescentes [Internet]. 2018. Disponible en: https://peru.unfpa.org/es/publications/ resultados-de-supervisi\%C3\%B3n-defensorial-servicios-de-saluddiferenciados-para

16. Ministerio de Salud del Perú. Guía Técnica para la Evaluación de la Satisfacción del Usuario Externo en los Establecimientos y Servicios Médicos de Apoyo [Internet]. 2012. Disponible en: http: / /bvs.minsa. gob.pe/local/MINSA/2252.pdf

17. Dagnew T, Tessema F, Hiko D. Health service utilization and reported satisfaction among adolescents in Dejen District, Ethiopia: a crosssectional study. Ethiop J Health Sci. 2015; 25(1): 17-28.

18. Khuzaiyah S, Muthoharoh A, Chabibah N. Satisfaction and motivation of adolescents attending a comprehensive teenage health services of Nasyiatul Aisyiyah. KnE Life Sciences. 2019; 4(13): 304-15.

19. Poccorpachi D. Proceso de atención de consulta externa y satisfacción del usuario adolescente en el área de obstetricia Hospital de Huaycán 2019 [Tesis de posgrado]. Lima: Universidad César Vallejo. Facultad de Medicina; 2019.

20. Awang H, Ab Rahman A, Sukeri S, Hashim N, Nik Abdul Rashid NR. Adolescent-friendly health services in primary healthcare facilities in Malaysia and its correlation with adolescent satisfaction level. Int J Adolesc Youth. 2020, 25(1): 551-61.

21. Chandra-Mouli V, Chatterjee S, Bose K. Do efforts to standardize, assess and improve the quality of health service provision to adolescents by government-run health services in low and middle income countries, lead to improvements in service-quality and service-utilization by adolescents?. Reprod Health. 2016; 13(10).

\section{Correspondencia:}

Danna Maribel Obregón Morales

Dirección: Av. San Antonio 161 - Villacanta, Independencia. Lima, Perú.

Teléfono: 955116511

Correo electrónico: danna.obregon@unmsm.edu.pe

Recibido: 11 de septiembre de 2020

Evaluado: 18 de octubre de 2020 Aprobado: 30 de diciembre de 2020

( $)$ La revista. Publicado por Universidad de San Martín de Porres, Perú. (c) BY Licencia de Creative Commons Artículo en acceso abierto bajo términos de Licencia Creative Commons Atribución 4.0 Internacional. (http://creativecommons.org/licenses/by/4.0/)

ORCID iDs

Danna Maribel Obregón-Morales ㄴ https://orcid.org/0000-0001-8582-804X Giovana Gladys Pante Salas C https://orcid.org/0000-0001-6666-6479

John Barja-Ore

- https: / / orcid.org/0000-0002-9455-0876

Andrea Mera-Yauri 